PROCEEDINGS OF THE

AMERICAN MATHEMATICAL SOCIETY

Volume 125, Number 1, January 1997, Pages 129-136

S 0002-9939(97)03523-5

\title{
SOBOLEV TYPE THEOREMS FOR AN OPERATOR WITH SINGULARITY
}

\author{
SHUJI WATANABE
}

(Communicated by Palle E. T. Jorgensen)

\begin{abstract}
Spaces of Sobolev type are discussed, which are defined by the operator with singularity: $\mathcal{D}=d / d x-(c / x) R$, where $R u(x)=u(-x)$ and $c>$ 1. This operator appears in a one-dimensional harmonic oscillator governed by Wigner's commutation relations. Smoothness of $u$ and continuity of $u / x^{\beta}$ $(\beta>0)$ are studied where $u$ is in each space of Sobolev type, and results similar to Sobolev's lemma are obtained.

The proofs are carried out based on a generalization of the Fourier transform. The results are applied to the Schrödinger equation.
\end{abstract}

\section{INTRODUCTION AND THE MAIN RESUlTS}

This paper is concerned with spaces of Sobolev type defined by the operator with singularity:

$$
\mathcal{D}=\frac{d}{d x}-\frac{c}{x} R, \quad R u(x)=u(-x)
$$

with a real constant $c$ specified later. The operator $p=-i \mathcal{D}$, called the momentum operator, appears in a one-dimensional harmonic oscillator governed by Wigner's commutation relations [7]:

$$
i p=[x, H] \text { and }-i x=[p, H],
$$

where the Hamiltonian is of the classical form

$$
H=\frac{1}{2}\left(p^{2}+x^{2}\right) \text {. }
$$

Assuming that $x$ is the multiplication by $x$, Yang [8] derived the above expression for $\mathcal{D}$. When $c=0$, Wigner's commutation relations are reduced to the canonical commutation relations in quantum mechanics and $\mathcal{D}$ becomes the differential operator $d / d x$.

To begin with, we define spaces of Sobolev type in terms of $\mathcal{D}$ instead of $d / d x$. Note that for $u \in C^{m}\left(\mathbf{R}^{1}\right), \mathcal{D}^{k} u(k=1,2, \cdots, m)$ is well defined at $x \neq 0$.

Received by the editors January 30, 1995 and, in revised form, May 31, 1995.

1991 Mathematics Subject Classification. Primary 46E35, 47B25, 81Q10.

Key words and phrases. Sobolev type theorem, operator with singularity, Schrödinger equation, self-adjointness. 
Definition. The space $\mathcal{C}^{m *}\left(\mathbf{R}^{1}\right)$ is the set of all $u \in C^{m}\left(\mathbf{R}^{1}\right)$ satisfying

$$
|u|_{m}=\sqrt{\sum_{k=0}^{m} \int_{-\infty}^{\infty}\left|\mathcal{D}^{k} u(x)\right|^{2} d x}<\infty .
$$

The space of Sobolev type $\mathcal{H}^{m}\left(\mathbf{R}^{1}\right)$ is the completion of $\mathcal{C}^{m *}\left(\mathbf{R}^{1}\right)$ under the norm $|\cdot|_{m}$.

Remark. When $c=0$, our space $\mathcal{H}^{m}\left(\mathbf{R}^{1}\right)$ coincides with the Sobolev space $H^{m}\left(\mathbf{R}^{1}\right)$.

The purpose of this paper is to prove the following theorems, and then to apply them to the Schrödinger equation.

Theorem 1. Suppose $c>1$. Let $\Omega$ be a bounded open interval of $\mathbf{R}^{1}$. Then, for $u \in \mathcal{H}^{m}\left(\mathbf{R}^{1}\right)$, there is an element $v \in C^{\alpha}(\Omega)$ such that $u(x)=v(x)$ a.e. in $\Omega$, where

$$
\alpha= \begin{cases}m-1 & (c=2 k), \\ \min (m-1, c-1) & (c=2 k+1), \\ \min (m-1,[c]) & (\text { otherwise })\end{cases}
$$

with $k \in \mathbf{N}(\mathbf{N}=\{1,2,3, \cdots\})$.

Theorem 2. Let $c, u$ and $v$ be as in Theorem 1. Then $v / x^{\beta}$ is continuous on $\Omega$, where

with $k \in \mathbf{N}$.

$$
\beta= \begin{cases}\min (m-1, c) & (c=2 k), \\ \min (m-1, c-1) & (c=2 k+1), \\ \min (m-1,[c]) & (\text { otherwise })\end{cases}
$$

Since the singular term $1 / x$ appears in the expression for $\mathcal{D}$, we mainly deal with the case where $\Omega$ contains the origin $x=0$. We remark that the relation $\alpha=m-1$ holds not only for $c=0$ (Sobolev's lemma) but also for $c=2 k$. See e.g. Yosida $\left[9\right.$, pp.174-175] for Sobolev's lemma. One may expects that $v / x^{\beta}$ is singular at the origin $x=0$. But, under the condition of Theorem 2, it remains continuous at the origin. As is well known, the Fourier transform plays an important role in the proof of Sobolev's lemma. In like manner we will prove our theorems using such a transform. This one, called the Bessel transform, is introduced in the study of the self-adjointness of the momentum operator $p=-i \mathcal{D}$ and the Hamiltonian $H=\left(p^{2}+x^{2}\right) / 2$ [4]. When $c=0$, the Bessel transform coincides with the Fourier transform, and hence it is regarded as a generalization of the Fourier transform.

\section{Preliminaries}

We summarize some results obtained previously in this direction. The Bessel transform $B$ is a unitary operator from $L^{2}\left(\mathbf{R}^{1}\right)$ onto itself [4, Theorem 3.2]:

$$
\|B u\|=\|u\|, \quad u \in L^{2}\left(\mathbf{R}^{1}\right)
$$

with $L^{2}$-norm $\|\cdot\|$. Moreover, we have

$$
B u(y)=\lim _{L \rightarrow \infty} \int_{-L}^{L} \overline{\varphi(x y)} u(x) d x,
$$

$$
B^{*} u(x)=\underset{L \rightarrow \infty}{\operatorname{li} . m .} \int_{-L}^{L} \varphi(x y) u(y) d y
$$


for $u \in L^{2}\left(\mathbf{R}^{1}\right)[4$, Theorem 3.4]. Here

$$
\varphi(x y)=\frac{\sqrt{|x y|}}{2}\left\{J_{c-1 / 2}(|x y|)+i \operatorname{sgn}(x y) J_{c+1 / 2}(|x y|)\right\}
$$

with $J_{\nu}$ being the Bessel function. When $c=0, \varphi(x y)=e^{i x y} / \sqrt{2 \pi}$ and the Bessel transform coincides with the Fourier transform. So the Bessel transform is regarded as a generalization of the Fourier transform. Ohnuki and Kamefuchi [3, pp.289-296] are the first to obtain the function $\varphi$ in the study of the eigenvalue problem of the momentum operator $p$ :

$$
p \varphi=y \varphi .
$$

The multiplication by $y$ is self-adjoint on the set $D(y)=\left\{u(y): u, y u \in L^{2}\left(\mathbf{R}^{1}\right)\right\}$. Moreover, the self-adjointness of $p$ is proved in [4] and [6]. With the aid of the Bessel transform, they are shown to be unitarily equivalent to each other $[4$, Proposition $4.2]$, i.e.,

$$
y=B p B^{*} .
$$

For unitary equivalence, see e.g. Goldstein [2, p.94].

The following are simple consequences of (2.3). Let us recall here that $c>1$.

$$
\begin{gathered}
\varphi(z)= \begin{cases}O\left(|z|^{c}\right) & (z \rightarrow 0) \\
O(\cos z) & (|z| \rightarrow \infty)\end{cases} \\
|\varphi(z)| \leq \text { constant } \cdot|z|^{c} \quad \text { for } z \text { in each bounded interval of } \mathbf{R}^{1} .
\end{gathered}
$$

Let us regard $\varphi(\cdot y)$ as a function of $x \in \Omega$ with $y \in \mathbf{R}^{1}$. Then :

$$
\varphi(\cdot y) \in C^{\gamma}(\Omega)
$$

where

with $k \in \mathbf{N}$.

$$
\gamma= \begin{cases}\infty & (c=2 k) \\ c-1 & (c=2 k+1) \\ {[c]} & \text { (otherwise) }\end{cases}
$$

\section{Proofs of Theorems 1 And 2}

Let us note that the momentum operator $p$ is self-adjoint on the set $D(p)=$ $H^{1}\left(\mathbf{R}^{1}\right) \cap D(1 / x)$ when $c>1[6$, Theorem 1]. Here $D(1 / x)=\{u(x): u, u / x \in$ $\left.L^{2}\left(\mathbf{R}^{1}\right)\right\}$. Then the operator $p^{m}$ is also self-adjoint on $D\left(p^{m}\right)=\left\{u(x): u, p u, p^{2} u\right.$, $\left.\cdots, p^{m-1} u \in D(p)\right\}$ for each positive integer $m$.

Lemma 1. Suppose $c>1$.

(i) If $v \in \mathcal{C}^{m *}\left(\mathbf{R}^{1}\right)$, then $v \in D\left(p^{k}\right)$ and $(-i \mathcal{D})^{k} v=p^{k} v(k=1,2, \cdots, m)$.

(ii) $\mathcal{H}^{m}\left(\mathbf{R}^{1}\right) \subset D\left(p^{m}\right)$.

Proof. We prove (i) by induction on $k$. An integration by parts gives that for $w \in D(p)$,

$$
\begin{aligned}
(-i \mathcal{D} v, w) & =-i\left(\frac{d v}{d x}, w\right)+i\left(\frac{c}{x} R v, w\right) \\
& =(v, p w)
\end{aligned}
$$


where $(\cdot, \cdot)$ denotes the inner product of $L^{2}\left(\mathbf{R}^{1}\right)$ defined by

$$
(u, v)=\int_{-\infty}^{\infty} u(x) \overline{v(x)} d x .
$$

Since $p$ is self-adjoint, $v \in D(p)$ and $-i \mathcal{D} v=p v$. Hence the lemma is true for $k=1$. Assume that it is also true for $k$. Then for $w \in D\left(p^{k+1}\right)$,

$$
\begin{aligned}
\left((-i \mathcal{D})^{k+1} v, w\right) & =\left((-i \mathcal{D})^{k} v, p w\right) \\
& =\cdots \cdots \\
& =\left(v, p^{k+1} w\right) .
\end{aligned}
$$

Since $p^{k+1}$ is also self-adjoint, $v \in D\left(p^{k+1}\right)$ and $(-i \mathcal{D})^{k+1} v=p^{k+1} v$. Thus (i) is true for $k+1$ and hence for $k=1,2, \cdots, m$. Combining (i) with the fact that the self-adjoint operator $p^{m}$ is closed yields (ii).

Lemma 2. If $c>1$, then $y^{k} B u \in L^{1}\left(\mathbf{R}^{1}\right)(k=0,1,2, \cdots, m-1)$ for $u \in$ $\mathcal{H}^{m}\left(\mathbf{R}^{1}\right)$.

Proof. For each $u \in \mathcal{H}^{m}\left(\mathbf{R}^{1}\right)$, there is a sequence $\left\{u_{n}\right\} \in \mathcal{C}^{m *}\left(\mathbf{R}^{1}\right)$ such that

$$
u_{n} \longrightarrow u, \mathcal{D}^{l} u_{n} \longrightarrow v^{l} \quad(l=1,2, \cdots, m) \text { in } L^{2}\left(\mathbf{R}^{1}\right),
$$

where $v^{l} \in L^{2}\left(\mathbf{R}^{1}\right)$. Since $B$ is a bounded operator (see (2.1)),

$$
B u_{n} \longrightarrow B u, B \mathcal{D}^{l} u_{n} \longrightarrow B v^{l} \quad \text { in } L^{2}\left(\mathbf{R}^{1}\right) \text {. }
$$

It follows from the preceding lemma combined with (2.5) that

$$
B \mathcal{D}^{l} u_{n}=(i y)^{l} B u_{n} .
$$

Choosing a subsequence $\left\{n^{\prime}\right\}$ of $\{n\}$ we therefore find

$$
B u_{n^{\prime}}(y) \longrightarrow B u(y),(i y)^{l} B u_{n^{\prime}}(y) \longrightarrow B v^{l}(y)
$$

for a.a. $y \in \mathbf{R}^{1}$. Hence

$$
(i y)^{l} B u(y)=B v^{l}(y) \quad \text { (a.e.), }
$$

where $l=1,2, \cdots, m$ and $B v^{l} \in L^{2}\left(\mathbf{R}^{1}\right)$. We are ready to evaluate the integral

$$
\int_{-\infty}^{\infty}\left|y^{k} B u(y)\right| d y \quad(k=0,1,2, \cdots, m-1) .
$$

By the Schwarz inequality,

$$
\int_{|y| \leq L}\left|y^{k} B u(y)\right| d y \leq \sqrt{\int_{|y| \leq L}\left|y^{k}\right|^{2} d y \int_{|y| \leq L}|B u(y)|^{2} d y}<\infty .
$$

On the other hand, with the help of (3.1),

$$
\begin{aligned}
\int_{|y|>L}\left|y^{k} B u(y)\right| d y & \leq \sqrt{\int_{|y|>L}\left|\frac{y^{k}}{\left(1+y^{2}\right)^{m / 2}}\right|^{2} d y \int_{|y|>L}\left|\left(1+y^{2}\right)^{m / 2} B u(y)\right|^{2} d y} \\
& <\infty .
\end{aligned}
$$

The lemma follows. 
We now turn to the expression (2.2). Let $\Omega$ be as in Theorem 1. Then for $u \in \mathcal{H}^{m}\left(\mathbf{R}^{1}\right)$,

$$
u(x)=\lim _{n \rightarrow \infty} \int_{-n}^{n} \varphi(x y) B u(y) d y \quad(\text { a.a. } x \in \Omega) .
$$

The function $y \mapsto\left|\varphi(x y) / \sqrt{1+y^{2}}\right|^{2}$ is integrable on $\mathbf{R}^{1}$, and so is the function $y \mapsto \varphi(x y) B u(y)$ by $(3.1)$. Hence

$$
u(x)=\int_{-\infty}^{\infty} \varphi(x y) B u(y) d y \quad(\text { a.a. } x \in \Omega) .
$$

Set

$$
v(x)=\int_{-\infty}^{\infty} \varphi(x y) B u(y) d y .
$$

Then $u(x)=v(x)$ a.e. in $\Omega$.

We will see that smoothness of $v$ together with continuity of $v / x^{\beta}$ follows from the two lemmas given just below.

Lemma 3. Suppose $c>1$. Let $\alpha$ be as in Theorem 1. Then $v$ is $\alpha$ times differentiable on $\Omega$.

Proof. Combining (2.4) with (2.8) yields

$$
\frac{\partial \varphi}{\partial x}=\left(\frac{c}{x y} R \varphi+i \varphi\right) y
$$

and

$$
\frac{\partial^{\alpha} \varphi}{\partial x^{\alpha}}=\left\{\sum_{k=0}^{\alpha} \frac{a_{k}^{(\alpha)} \varphi+b_{k}^{(\alpha)} R \varphi}{(x y)^{\alpha-k}}\right\} y^{\alpha}
$$

with $a_{k}^{(\alpha)}$ and $b_{k}^{(\alpha)}$ chosen appropriately. We formally differentiate $v \alpha$ times.

$$
\frac{d^{\alpha} v}{d x^{\alpha}}=\int_{-\infty}^{\infty} \frac{\partial^{\alpha} \varphi}{\partial(x y)^{\alpha}} y^{\alpha} B u(y) d y .
$$

The result follows from (2.6), (2.8) and Lemma 2.

A direct computation gives the following lemma.

Lemma 4. Suppose that $g: \Omega \times \mathbf{R}^{1} \rightarrow \mathbf{C}^{1}$ satisfies the following conditions:

(i) $|g(x, y)| \leq$ constant.

(ii) For each $L>0$, there is a number $K$ such that $|g(x, y)-g(a, y)| \leq$ $K|x-a|^{\beta}$, where $\beta>0$ and $|y| \leq L$.

Then the function $F$ defined by

$$
F(x)=\int_{-\infty}^{\infty} g(x, y) f(y) d y \quad\left(f \in L^{1}\left(\mathbf{R}^{1}\right)\right)
$$

is continuous at $x=a$.

We are now in a position to prove our theorems.

Proof of Theorem 1. By Lemma 3, it is enough to prove the continuity of $d^{\alpha} v / d x^{\alpha}$.

The case where $c=2 k \quad(k \in \mathbf{N})$. Note that $\alpha=m-1$. Thanks to Lemma 2, we have only to show that $\partial^{\alpha} \varphi / \partial(x y)^{\alpha}$ in (3.3) satisfies the conditions (i) and (ii) of Lemma 4. However, it follows immediately from (2.6) and (2.8). 


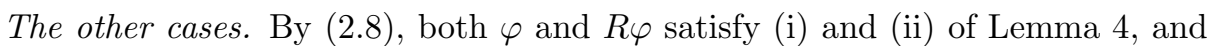
hence the functions

$$
\int_{-\infty}^{\infty} \varphi(x y) y^{k} B u(y) d y \quad \text { and } \quad \int_{-\infty}^{\infty} R \varphi(x y) y^{k} B u(y) d y \quad(k=0,1, \cdots, \alpha)
$$

are continuous on $\Omega$. Thus $d^{\alpha} v / d x^{\alpha}$ is shown to be continuous at $x \neq 0$. So it remains to show the continuity at the origin. Note that $d^{\alpha} v / d x^{\alpha}(0)=0$. $\partial^{\alpha} \varphi / \partial(x y)^{\alpha}$ clearly satisfies (i) of Lemma 4 by (2.6). Moreover, (2.7) gives

$$
\left|\frac{\partial^{\alpha} \varphi}{\partial(x y)^{\alpha}}\right| \leq \text { constant } \cdot \sum_{k=0}^{\alpha}\left(\left|a_{k}^{(\alpha)}\right|+\left|b_{k}^{(\alpha)}\right|\right)|x y|^{c-\alpha+k}
$$

where $c-\alpha+k>0$. Therefore, the condition (ii) is also satisfied. Thus $d^{\alpha} v / d x^{\alpha}$ is continuous at $x=0$.

This completes the proof of Theorem 1.

Proof of Theorem 2. By (3.2),

$$
\frac{v(x)}{x^{\beta}}=\int_{-\infty}^{\infty} \frac{\varphi(x y)}{(x y)^{\beta}} y^{\beta} B u(y) d y .
$$

Lemma 4 implies that $v$ is continuous on $\Omega$, and so it suffices to show the continuity of $v / x^{\beta}$ at $x=0$.

The case where $c=2 k$ and $c \leq m-1$. Note that $\beta=c$. By $2 k \leq m-1$, Lemma 2 implies that the integral

$$
\left[\frac{v}{x^{\beta}}\right]_{x=0}=\int_{-\infty}^{\infty} \frac{y^{2 k}}{2^{2 k+1 / 2} \Gamma(2 k+1 / 2)} B u(y) d y
$$

is well defined. Here $\Gamma$ denotes the gamma function. Then

$$
\frac{v(x)}{x^{\beta}}-\left[\frac{v(x)}{x^{\beta}}\right]_{x=0}=\int_{-\infty}^{\infty} G(x y) y^{2 k} B u(y) d y,
$$

where

$$
G(x y)=\frac{\varphi(x y)}{(x y)^{2 k}}-\frac{1}{2^{2 k+1 / 2} \Gamma(2 k+1 / 2)} .
$$

It follows from (2.6) that $G(x y)$ satisfies (i) of Lemma 4. Furthermore, since $|G(x y)| \leq$ constant $\cdot|x y|$, the condition (ii) is also satisfied. Thus $v / x^{\beta}$ is continuous at $x=0$.

The other cases. By (2.6), $\varphi /(x y)^{\beta}$ satisfies (i) of Lemma 4. Note that $\left[\varphi /(x y)^{\beta}\right]_{x=0}=0$. It follows from (2.7) that

$$
\left|\frac{\varphi(x y)}{(x y)^{\beta}}-0\right| \leq \text { constant } \cdot|x y|^{c-\beta} \text {. }
$$

Thus the condition (ii) is satisfied, and hence $v / x^{\beta}$ is continuous at $x=0$.

The proof of Theorem 2 is complete.

\section{Application to the Schrödinger equation}

As stated before, the momentum operator $p$ is self-adjoint on the set $D(p)=$ $H^{1}\left(\mathbf{R}^{1}\right) \cap D(1 / x)$ when $c>1$. Hence the operator $p^{2}$ is also self-adjoint on the set $D\left(p^{2}\right)=\{u \in D(p): p u \in D(p)\}$ when $c>1$. Moreover, it is shown that the Hamiltonian $H=\left(p^{2}+x^{2}\right) / 2$ is self-adjoint on the set $D(H)=D\left(p^{2}\right) \cap D\left(x^{2}\right)$ when $c>1\left[6\right.$, Theorem 2]; [4, Theorem 2.2]. Here $D\left(x^{2}\right)=\left\{u(x): u, x^{2} u \in L^{2}\left(\mathbf{R}^{1}\right)\right\}$. 
As an application of our main theorems we consider the following Schrödinger equation for a one-dimensional harmonic oscillator governed by Wigner's commutation relations:

$$
\left\{\begin{array}{l}
i \frac{\partial u}{\partial t}=\frac{1}{2}\left\{-\frac{\partial^{2}}{\partial x^{2}}+\frac{c}{x^{2}}(c-R)+x^{2}\right\} u \quad(=H u), \quad x \in \mathbf{R}^{1} \\
u(0, x)=u_{0}(x), \quad x \in \mathbf{R}^{1} .
\end{array}\right.
$$

We prepare two lemmas.

Lemma 5. If $c \geq 3 / 2$, then the operator $p^{2}$ is essentially self-adjoint on $C_{0}^{\infty}\left(\mathbf{R}^{1} \backslash\{0\}\right)$.

Proof. Let $A$ be the operator $p^{2}$ restricted to $C_{0}^{\infty}\left(\mathbf{R}^{1} \backslash\{0\}\right)$. Then

$$
A v=\left\{-\frac{d^{2}}{d x^{2}}+\frac{c(c-R)}{x^{2}}\right\} v \quad \text { for } v \in C_{0}^{\infty}\left(\mathbf{R}^{1} \backslash\{0\}\right) .
$$

Let us show $\{\operatorname{Ran}(i+A)\}^{\perp}=\{0\}$. If $u \in\{\operatorname{Ran}(i+A)\}^{\perp}$, then so is $u_{ \pm}=(u \pm R u) / 2$. Hence

$$
\left(u_{ \pm},\left\{i-\frac{d^{2}}{d x^{2}}+\frac{c(c \mp 1)}{x^{2}}\right\} v\right)=0 \quad \text { for } v \in C_{0}^{\infty}\left(\mathbf{R}^{1} \backslash\{0\}\right) .
$$

As long as $c \geq 3 / 2, c(c \mp 1) \geq 3 / 4$. So the following result is applicable: The operator $L(k)=-d^{2} / d x^{2}+k / x^{2}$ is essentially self-adjoint on $C_{0}^{\infty}\left(\mathbf{R}^{1} \backslash\{0\}\right)$ provided $k \geq 3 / 4$. See e.g. Reed and Simon [5, p.186]. Since the number $i$ is in the resolvent set of the self-adjoint operator $L(k)^{*}$, it follows that $u_{ \pm}=0$, and hence $u=0$. Thus $\{\operatorname{Ran}(i+A)\}^{\perp}=\{0\}$. A similar argument gives $\{\operatorname{Ran}(-i+A)\}^{\perp}=\{0\}$. Since each of the deficiency indices of $A$ is equal to 0 , the closure of $A$ becomes self-adjoint and coincides with $p^{2}$.

Lemma 6. If $c \geq 3 / 2$, then $D\left(p^{2}\right)=\mathcal{H}^{2}\left(\mathbf{R}^{1}\right)$.

Proof. By Lemma 1 (ii), it is enough to show $D\left(p^{2}\right) \subset \mathcal{H}^{2}\left(\mathbf{R}^{1}\right)$. But this follows immediately from Lemma 5 .

As mentioned above, the Hamiltonian $H$ is self-adjoint on the set $D(H)$. Therefore, $-i H$ generates a strongly continuous unitary group on $L^{2}\left(\mathbf{R}^{1}\right)$. We thus see that $u(t, \cdot)=\exp (-i t H) u_{0}, u_{0} \in D(H)$ is a unique solution of the Schrödinger equation (4.1). Note that the solution $u(t, \cdot)$ is in $D(H)$. From Lemma 6 and our main theorems we thus obtain the following.

Proposition 7. Let $c \geq 3 / 2$. Then, for each $u(t, \cdot)$, there is an element $v(t, \cdot) \in$ $C^{1}(\Omega)$ such that $u(t, x)=v(t, x)$ a.e. in $\Omega$ and the function $x \mapsto v(t, x) / x$ is continuous on $\Omega$.

Remark. For $c<-1$, results similar to our main theorems and our proposition are obtained as long as (2.3) is replaced with

$$
\varphi(x y)=\frac{\sqrt{|x y|}}{2}\left\{\operatorname{sgn}(x y) J_{-c-1 / 2}(|x y|)+i J_{-c+1 / 2}(|x y|)\right\} .
$$




\section{REFERENCES}

[1] S. Agmon, Lectures on elliptic boundary value problems, Van Nostrand, Princeton, 1965. MR 31:2504

[2] J. A. Goldstein, Semigroups of linear operators and applications, Oxford University Press, New York, 1985 / Clarendon Press, Oxford, 1985. MR 87c:47056

[3] Y. Ohnuki and S. Kamefuchi, Quantum field theory and parastatistics, University of Tokyo Press, Tokyo, 1982 / Springer-Verlag, Berlin, Heidelberg and New York, 1982. MR 85b:81001

[4] Y. Ohnuki and S. Watanabe, Self-adjointness of the operators in Wigner's commutation relations, J. Math. Phys. 33 (1992), 3653-3665. MR 93h:81065

[5] M. Reed and B. Simon, Methods of modern mathematical physics, vol. II, Fourier analysis, self-adjointness, Academic Press, New York, 1975. MR 58:12429b

[6] M. Watanabe and S. Watanabe, Self-adjointness of the momentum operator with a singular term, Proc. Amer. Math. Soc. 107 (1989), 999-1004. MR 90g:81035

[7] E. P. Wigner, Do the equations of motion determine the quantum mechanical commutation relations?, Phys. Rev. 77 (1950), 711-712. MR 90g:81035

[8] L. M. Yang, A note on the quantum rule of the harmonic oscillator, Phys. Rev. 84 (1951), 788-790. MR 13:804e

[9] K. Yosida, Functional analysis, 6th ed., Springer-Verlag, Berlin, Heidelberg and New York, 1980. MR 82i: 46002

Department of Mathematics, Toyota National College of Technology, Eisei-Cho 2-1, TOYOTA-SHI 471, JAPAN 